\title{
Effects of obesity and 10 weeks metformin treatment on liver steatosis
}

\author{
REZA HAKKAK $^{1-3}$, SHANNON ROSE $^{2,3}$, BEVERLY SPRAY $^{3}$, MELISA KOZACZEK $^{1,3}$ and SOHEILA KOROURIAN ${ }^{4}$ \\ Departments of ${ }^{1}$ Dietetics and Nutrition, and ${ }^{2}$ Pediatrics, University of Arkansas for Medical Sciences, Little Rock, AR 72205; \\ ${ }^{3}$ Arkansas Children's Research Institute, Little Rock, AR 72202; \\ ${ }^{4}$ Department of Pathology, University of Arkansas for Medical Sciences, Little Rock, AR 72205, USA
}

Received October 2, 2020; Accepted February 8, 2021

DOI: $10.3892 /$ br.2021.1425

\begin{abstract}
Non-alcoholic fatty liver disease (NAFLD) is the leading cause of liver disease in adolescents and adults, and the risk of developing NAFLD increases with obesity. In the present study, it was shown that obesity increased fatty liver (steatosis) using an obese Zucker rat model. Metformin is an oral anti-hyperglycemic agent approved by the FDA for treatment of type 2 diabetes in adults and children $>10$ years of age. There is insufficient evidence regarding the effects of metformin on pediatric liver steatosis. Thus, in the present study, the effects of 10 weeks metformin treatment on liver steatosis and related serum markers for liver damage was assessed. Lean and obese ( $n=16$ per group) 5-week old female Zucker rats were provided an AIN-93 G diet for 8 weeks to induce NAFLD, and then rats were randomly assigned to 4 groups (8 rats/group): i) lean without metformin (LC), ii) lean + metformin (LM), iii) obese without metformin (OC), and iv) obese + metformin (OM). Rats were provided ad libitum access to the diet containing metformin $(1 \mathrm{~g}$ metformin per $\mathrm{kg}$ of food). Rats were weighed twice weekly and were sacrificed 10 weeks later. Serum was collected to measure the levels of aspartate aminotransferase (AST), alanine aminotransferase (ALT), leptin and adiponectin. Livers were collected for histological analysis. The results showed that obese rats gained significantly more weight than lean rats in both the control and metformin treatment groups $(\mathrm{P}<0.001)$. OM treated rats exhibited a lower degree of liver steatosis compared with the OC rats $(\mathrm{P}<0.04)$. There were no significant differences in serum ALT levels between the groups. However, obesity significantly increased serum AST levels in both the control and metformin treatment groups $(\mathrm{P}=0.01)$. The ratio of leptin to adiponectin was increased in obese compared with the lean rats in both the control and metformin treatment groups $(\mathrm{P}<0.0001)$. There
\end{abstract}

Correspondence to: Dr Reza Hakkak, Department of Dietetics and Nutrition, University of Arkansas for Medical Sciences, 4301 West Markham Street, Mail Slot 627, Little Rock, AR 72205, USA

E-mail:RHakkak@uams.edu

Key words: obesity, metformin, liver steatosis, Zucker rats was no effect of metformin on serum biomarkers. In summary, short-term metformin treatment decreased liver steatosis but did not affect the serum markers of liver steatosis.

\section{Introduction}

Obesity has been classified as an epidemic in the United States for $>30$ years. Recent data (2020) from the Centers for Disease Control (CDC) place the prevalence of obesity in US adults at $42.4 \%$ in $2017-2018$, up from $30.5 \%$ in $1999-2000$, while the prevalence of severe obesity has increased from 4.7 to $9.2 \%$ during the same period (1). Worldwide, $>1.9$ billion adults are overweight, and >600 million adults are obese (2). In the USA, childhood obesity affects $\sim 12.5$ million children and teens. Data in a nationally representative study of US children and adolescents aged 2-19 years showed that the prevalence of obesity in 2011-2014 was $17.0 \%$ and that of extreme obesity was $5.8 \%(3,4)$. Obesity has been associated with increased morbidities and mortality rates, including diabetes, cardiovascular disease, several types of cancer and liver steatosis (5). Importantly, the incidence of these life-threatening comorbidities increases with the duration of obesity, and therefore, age.

Non-alcoholic fatty liver disease (NAFLD), the major cause of abnormal liver function in the US and worldwide, is often associated with obesity and diabetes (5). The mortality rates in individuals with NAFLD is significantly higher than in the general population, with liver-related complications being a common cause of death (6). An estimated 70 million adults and 7 million US children have NAFLD. Amongst the children with obesity, NAFLD is present in $33-58 \%$ of cases, and it is now the most common cause of chronic liver disease in the pediatric population (7). Data from our laboratory using the obese Zucker rat model suggest that obesity serves an important role in promotion of liver steatosis (NAFLD) $(8,9)$.

Metformin is a first line oral anti-hyperglycemic agent approved by the FDA in 1994 to treat type 2 diabetes in adults and children $>10$ years of age. Although it has been proven to be safe after decades of use, its exact mechanisms of action remains unclear and contested (10). It is not metabolized and it is excreted by the kidneys and bile (11). In the liver, it has been shown to inhibit complex I of the mitochondrial respiratory chain, and to activate AMP-activated protein kinase, 
processes that have been related to its ability to inhibit hepatic lipogenesis and gluconeogenesis, increasing hepatic insulin sensitivity, indirectly lowering circulating glucose and insulin levels $(10,12,13)$. These findings have encouraged the research of metformin as a pharmacological treatment for NAFLD. Several investigators have used different animal models and doses of metformin to study its effect on liver steatosis. There have been positive reports of metformin reducing liver steatosis (14-16), but these are not conclusive, and additionally negative studies have also been published (17-19). There is insufficient evidence regarding the effects of metformin in pediatric obesity. There are very few published data on the effects of metformin on liver steatosis in the adolescent population; therefore, the role of metformin on protection from NAFLD in an adolescent model was investigated. The major objectives of this study were to investigate the effects of obesity and short-term metformin treatment on i) body weight, ii) liver steatosis score and iii) serum aspartate aminotransferase (AST), alanine aminotransferase (ALT) and leptin and adiponectin levels. Lean and obese female Zucker rats were placed on a control diet for 8 weeks to induce NAFLD, and then both lean and obese rats were randomly placed on a diet with or without metformin (1 $\mathrm{g}$ metformin per $\mathrm{kg}$ of food) for 10 weeks. Obese Zucker rats were used as the model for early adolescent obesity related diseases (20).

\section{Materials and methods}

Experimental design. The animal protocols used in the present study were approved by the Institutional Animal Care and Use Committee (IACUC) at the University of Arkansas for Medical Sciences in 2018 (approval no. 3882).

A total of 32, 5-week-old female Zucker rats (16 obese fa/fa and 16 lean) were purchased from Envigo. The rats were genotypically identified fa/fa and lean/lean rats at 24 days of age. Upon receipt, the rats were housed 1 per cage with ad libitum access to water and a semi-purified diet similar to AIN-93G diet, containing casein $(20 \% \mathrm{w} / \mathrm{w} /$ protein) a dietary source of protein (Envigo) for 8 weeks to induce NAFLD $(8,9)$. After 8 weeks, lean and obese rats were randomly assigned to one of the following four groups (8 rats/group): i) lean without metformin (LC), ii) lean with metformin (LM), iii) obese without metformin (OC), and iv) obese with metformin (OM). Metformin was mixed with the AIN-93G diet at $1 \mathrm{~g}$ metformin per $\mathrm{kg}$ of food. Rats were weighed twice per week. All rats were sacrificed 10 weeks post-metformin treatment, using $\mathrm{CO}_{2}(30 \%)$ prior to decapitation. Livers and blood samples were collected following euthanasia. Liver samples and serum were stored at $-80^{\circ} \mathrm{C}$ for subsequent experiments.

Livers were removed and weighed individually. Per each lobe of the liver, two $3-\mathrm{mm}$ sections were fixed in $10 \%$ buffered formalin at room temperature for 2 days for histological examination. Liver sections were cut $(5 \mu \mathrm{m})$ and stained with hematoxylin and eosin (H\&E) for $45 \mathrm{~min}$ at room temperature. A board-certified anatomic pathologist evaluated the H\&E stained sections of the livers, and they were blinded to the conditions. The presence and extent of microvesicular and macrovesicular steatosis was examined. Steatosis was semi-quantitatively scored between 0 and 4 as follows: 0 , no steatosis; 1 , steatosis in $<25 \%$ of the hepatocytes; 2 , steatosis

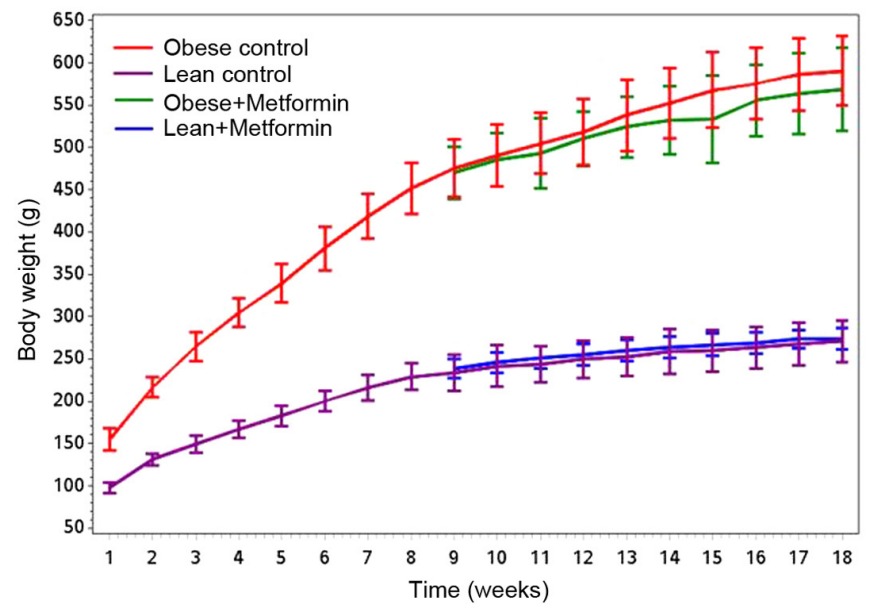

Figure 1. Mean body weight \pm standard deviation of lean and obese rats during the 8 weeks on the control diet prior to metformin treatment, and in the 10 weeks after metformin treatment.

in $25-50 \%$ of the hepatocytes; 3 , steatosis in $51-75 \%$ of the hepatocytes; and 4, steatosis in $>75 \%$ of the hepatocytes as previously reported $(8,9)$.

Serum analysis. Blood ( $2 \mathrm{ml})$ was collected immediately after decapitation into $50 \mathrm{ml}$ centrifuge tubes, allowed to clot, and centrifuged at $2,000 \mathrm{x} \mathrm{g}$ for $10 \mathrm{~min}$ at room temperature to separate serum. Serum was aliquoted and stored at $-80^{\circ} \mathrm{C}$ for further analyses. Leptin and adiponectin levels were measured using ELISA kits (cat. nos. EZRL-83K and EZRADP-62K, respectively; EMD Millipore) according to the manufacturer's protocol. Serum AST and ALT concentrations were analyzed on an RX Daytona Clinical Analyzer (Randox).

Statistical analysis. Data on all outcome variables were assessed for normality using the Shapiro-Wilk test and box-and-whisker plots. The assumption of equal variance was verified using a Levene's test. In cases where the assumption of normality or equal variance was violated, the Welch's test statistic was used. Data are presented as the mean \pm standard deviation. To determine if outcome variables differed between lean and obese rats and with or without metformin, a general linear model procedure was employed with treatment as the primary effector. If there was a significant primary effect of treatment, the statistical differences among the treatments were analyzed using contrast statements in the SAS GLM procedure. Multiple comparisons amongst means were adjusted using Tukey's honestly significant difference tests. $\mathrm{P}<0.05$ was considered to indicate a statistically significant difference. Statistical analysis was performed in SAS version 9.4 (SAS Institute).

\section{Results}

Body weight. Mean body weight in grams at the beginning of the experiment was $97.8 \pm 6.5$ and $154.8 \pm 13.3$ for LC and OC rats, respectively. Table I presents the body weights at the end of the 18-week experiment. Fig. 1 shows that obese rats gained significantly more weight $(\mathrm{P}<0.001)$ compared with the lean rats for both control and metformin treatment groups, and there was no significant difference between $\mathrm{OC}$ vs. OM groups 
Table I. Effects of obesity and metformin treatment on the final BW, liver weight and liver weight as a percentage of BW.

\begin{tabular}{|c|c|c|c|c|c|c|c|c|}
\hline \multirow[b]{2}{*}{ Parameter } & \multicolumn{4}{|c|}{ Mean standard \pm deviation } & \multicolumn{4}{|c|}{ P-value } \\
\hline & $\mathrm{LC}$ & LM & $\mathrm{OC}$ & $\mathrm{OM}$ & LC vs. LM & LC vs. OC & LM vs. OM & OC vs. OM \\
\hline Final BW & $268.0 \pm 26.3$ & $278.0 \pm 13.8$ & $598.0 \pm 41.4$ & $573.0 \pm 48.1$ & 0.521 & $<0.001^{\mathrm{a}}$ & $<0.001^{\mathrm{a}}$ & 0.207 \\
\hline Liver weight, g & $8.1 \pm 1.3$ & $8.8 \pm 1.0$ & $35.5 \pm 4.6$ & $34.9 \pm 4.3$ & 0.652 & $<0.001^{\mathrm{a}}$ & $<0.001^{\mathrm{a}}$ & 0.743 \\
\hline Liver weight, \%BW & $3.0 \pm 0.3$ & $3.1 \pm 0.3$ & $3.0 \pm 1.0$ & $6.1 \pm 0.6$ & 0.618 & $<0.001^{\mathrm{a}}$ & $<0.001^{\mathrm{a}}$ & 0.710 \\
\hline
\end{tabular}

${ }^{\text {a }}<<0.001$. BW, body weight; LC, lean control; LM, lean + metformin; OC, obese control; OM, obese + metformin.

A

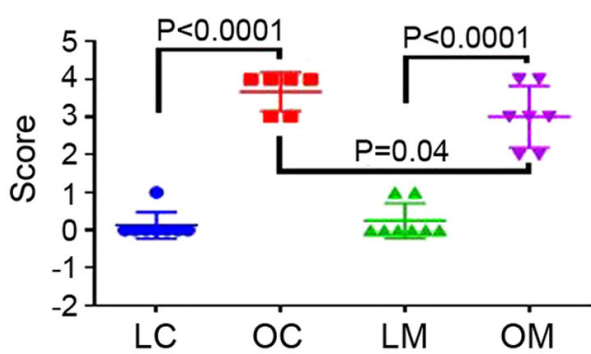

B

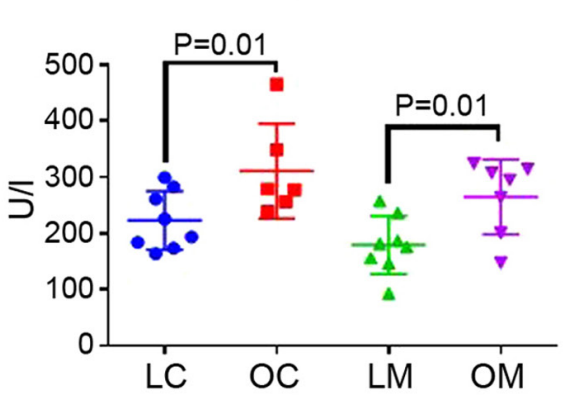

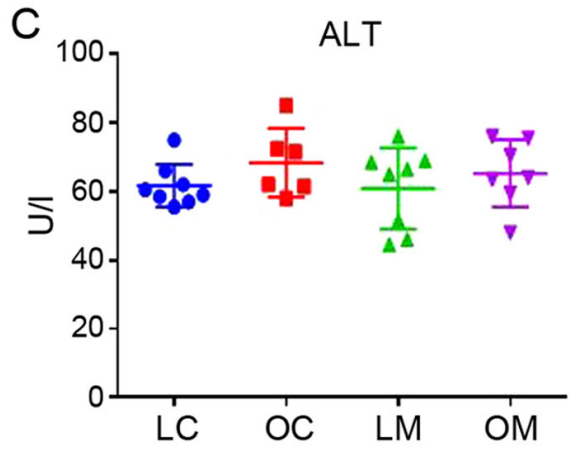

Figure 2. Effects of obesity and metformin treatment on parameters of liver function. Effects of obesity and metformin treatment on (A) liver steatosis score, (B) serum AST and (C) serum ALT levels. AST, aspartate aminotransferase; ALT, alanine aminotransferase; LC, lean control; LM, lean + metformin; OC, obese control; OM, obese + metformin.

$(\mathrm{P}=0.20)$. Final body weights differed significantly between the $\mathrm{LC}$ and $\mathrm{OC}$ rats as well as between the LM and OM rats $(\mathrm{P}<0.0001$; Table I). There was no significant difference in the final body weights between LC and LM rats or between OC and $\mathrm{OM}$ rats. The liver weights in $\mathrm{g}$ and as a percentage of final body weight are presented in Table I.

Liver weights and histological analysis. The liver weights in $\mathrm{g}$ and as a percentage of final body weight is presented in Table I. Liver weights in $g$ and as a percentage of final body weight were significantly higher in obese rats compared with the lean rats in both the control and metformin treated groups $(\mathrm{P}<0.0001)$. Steatosis scores are presented in Fig. 2A. Steatosis scores were significantly elevated in obese rats compared with the lean rats in both control (OC) and metformin (OM) treated groups $(\mathrm{P}<0.0001)$. In addition, rats in the OM group had lower levels of liver steatosis compared to the OC group ( $\mathrm{P}<0.04$; Fig. 2A). Representative photomicrographs of liver parenchyma of lean and obese rats with and without metformin treatment are shown in Fig. 3.

Serum measurements. Figs. 2 and 4 show the serum levels of ALT, AST, leptin and adiponectin. Serum AST levels were significantly elevated in obese rats compared with the lean rats in both the control and metformin treatment groups $(\mathrm{P}=0.01)$; however, serum ALT levels did not differ between groups. Leptin $(\mathrm{P}<0.0001)$, adiponectin $(\mathrm{P}=0.01)$ and the ratio of leptin to adiponectin $(\mathrm{P}<0.0001)$ were all increased in obese rats compared with the lean rats in both the control and metformin treatment groups. The leptin/adiponectin ratio is an important marker of insulin resistance in obesity (20). There were no effects of metformin on any of the serum markers.

\section{Discussion}

To investigate the role of metformin and obesity on liver steatosis, the Zucker rat (fa/fa) model was used, which is the most widely used model for obesity related research. Obesity in the Zucker rat is inherited as an autosomal recessive trait caused by a mutation in the leptin receptor gene, such that Zucker rats become noticeably obese by the age of 3 to 5 weeks, and by 14 weeks, $>40 \%$ of their body is composed of lipids (21). Obese Zucker rats develop hyperinsulinemia and insulin resistance before they develop obesity-associated, non-insulin-dependent diabetes mellitus in a manner similar to that in humans, making them an excellent model for investigating the relationship between obesity and liver steatosis. Lean Zucker rats, in contrast, exhibit normal metabolic function and are considered ideal controls $(22,23)$. In addition, this animal model develops hepatic steatosis due to dysregulated metabolic gene expression in the liver.

NAFLD is the most commonly observed liver problem in obese pediatric and adult populations in the US as well as worldwide, and it is primarily managed through lifestyle changes, as with obesity and type 2 diabetes. Success with lifestyle changes is hampered by patient adherence, particularly in the pediatric population, and alternative therapeutics are thus required. In our previous study, it was shown that using the obese Zucker rat model, obesity increased body weight, and this resulted in an increase in liver steatosis compared with lean rats $(8,9,21,24)$. 

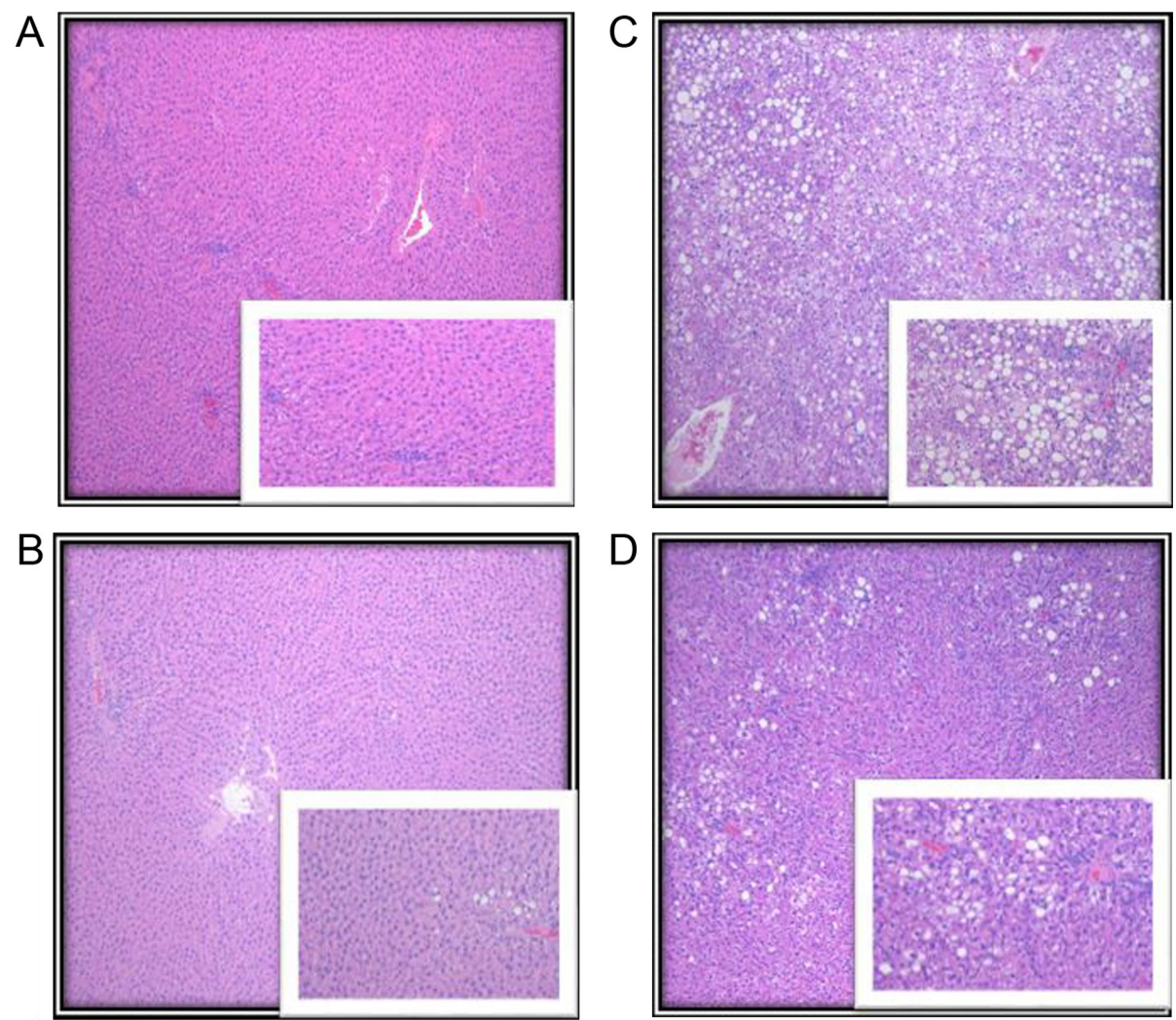

Figure 3. Representative images of liver parenchyma of the lean and obese rats with and without metformin treatment. (A) LC, showing complete preservation of the architecture with no evidence of fatty changes, as shown in the higher magnification insert. (B) LM, showing complete preservation of the architecture with minimal steatosis seen in $<2 \%$ of the hepatocytes, predominantly within zone 3 (periportal region). (C) OC, preservation of overall architecture with macrosteatosis and microsteatosis seen in $>75 \%$ of hepatocytes, which involved all three zones (central, mid and periportal region). (D) OM, preservation of overall architecture with steatosis seen in $25 \%$ of hepatocytes, macrovesicular type, predominantly in the periportal region. Lower right inserts show higher magnification of the zone involved in steatosis. Original magnification, x40; insert, x100. LC, lean control; LM, lean + metformin; OC, obese control; OM, obese + metformin.

A

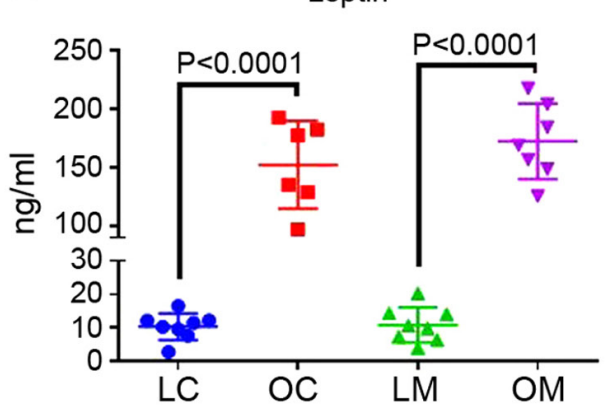

B

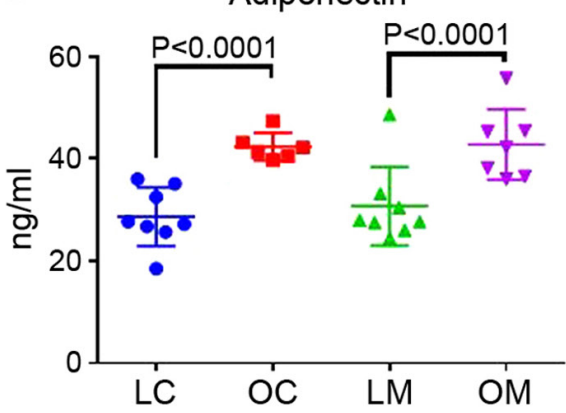

C

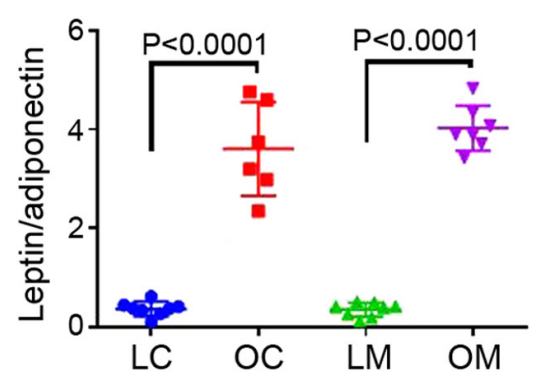

Figure 4. Effects of obesity and metformin treatment on the serum lipid profile. Effect of obesity and metformin treatment on serum (A) leptin, (B) adiponectin and (C) leptin/adiponectin levels. LC, lean control; LM, lean + metformin; OC, obese control; OM, obese + metformin.

However, the effect of short-term metformin treatment on liver steatosis and the related liver enzymes has not been previously assessed, to the best of our knowledge.

In the present study, it was shown that obese rats gained significantly more weight than lean rats, and metformin treatment had no effect on weight gain. Furthermore, liver steatosis was significantly higher in obese rats compared with the lean rats, and metformin treatment reduced liver steatosis. This result was further supported by the changes in serum AST levels. The leptin to adiponectin ratio was increased in obese rats compared with the lean rats, and metformin treatment had no effect on the levels of these serum biomarkers. Metformin was previously shown to reduce liver steatosis in ob/ob leptin deficient mice, and to also reduce hepatic TNF expression (14). One of the first pilot studies in humans to assess metformin treatment on liver steatosis showed a promising increase in insulin sensitivity, reduction of ALT levels and a reduction in the volume of the liver (15). In addition, metformin inhibits inflammatory signaling, which in turn suppresses the production of proinflammatory cytokines in the liver tissues (25). Cyclooxygenase-2 (COX-2) is considered to be partly responsible for the obesity-related inflammation in diabetes and fatty 
liver. A COX-2 inhibitor was found to exert a synergistic beneficial effect with metformin on obesity-associated metabolic and cardiovascular disorders in male Sprague-Dawley rats fed a high-fat diet (26). Metformin improved hepatic insulin receptor substrate 2 and PI3K/Akt signaling in insulin-resistant rats of a non-alcoholic steatohepatitis (NASH) and cirrhosis model, where the pathophysiological appearance of the liver was largely improved by treatment with metformin, and a decrease in lipid and collagen accumulation was observed in the liver tissues (16).

There is insufficient evidence regarding the effects of short-term metformin treatment on pediatric obesity and liver steatosis. Several clinical trials have identified modest improvements following metformin treatment in insulin sensitivity in obese children with normal glucose tolerance (27-29), as well as a decrease in the BMI of obese adolescents (30). In addition, metformin appears to improve lipid profiles in obese children (31,32). El-Lakkany et al (33) found that the co-administration of metformin and $\mathrm{N}$-acetylcysteine, the precursor of the antioxidant glutathione, paired with dietary control improved the biochemical and histological manifestations in rats with NAFLD (33). Additionally, the concomitant administration of fish oil with metformin regulates the expression of genes involved in lipid metabolism in a diabetic rat model, exerting potentially beneficial effects (34).

It has been reported that short-term metformin treatment has beneficial effects on lowering blood lipid levels and protecting hepatocytes from lipid accumulation (2,7-9); however, several studies with long-term metformin treatment did not show histological protection of hepatic tissue $(21,22,24)$.

Studies have used the Zucker diabetic fatty (ZDF) rat as a diabetic model to investigate the effects of long-term metformin treatment. As well as different doses of metformin on liver steatosis. Sui et al (35) placed ZDF rats on either vehicle or metformin treatment $(50 \mathrm{mg} / \mathrm{kg}$ body weight) for 6 months. They reported that metformin treatment reduced blood glucose, but this did not prevent the development of liver steatosis and dysregulated blood lipid profiles. Chen et al (36) used Sprague Dawley rats on a high fat diet to induce obesity and type 2 diabetes mellitus, and were placed on either a low-dose $(100 \mathrm{mg} / \mathrm{kg})$ or high-dose $(200 \mathrm{mg} / \mathrm{kg})$ metformin derivative (MD568), or metformin (200 mg/kg) for 8 weeks. They reported that the new metformin derivative MD568 significantly reduced plasma glucose, insulin, total cholesterol, triglyceride and low-density lipoprotein cholesterol levels. Additionally, MD568 treatment also improved the insulin resistance of obese type 2 diabetes mellitus model rats.

There are potential limitations in the present study, including the sample size and the length of the experiment. A larger sample size and a longer period under treatment with metformin may strengthen the weight/power of the data. Nevertheless, the results show that metformin is a suitable candidate for further study on its effects in reducing liver steatosis in the pediatric population.

In conclusion, it was shown that 10 weeks metformin treatment in obese rats reduced liver steatosis, but had no effects on the levels of serum markers. It is hypothesized that a longer treatment period may be required for the metformin treatment to exert a significant effect on the levels of liver damage markers.

\section{Acknowledgements}

We would like to thank Ms. Sheena Joyner (Department of Dietetics and Nutrition at University of Arkansas for Medical Sciences) for her valuable assistance in preparation of this manuscript.

\section{Funding}

The present study was supported by funding from the Arkansas Bioscience Institute for funding and the National Institute of General Medical Sciences of the National Institutes of Health (grant no. 5P20GM109096).

\section{Availability of data and materials}

The datasets used and/or analyzed in the present study are all included in the published article.

\section{Authors' contributions}

RH designed the study, and participated in the collection of data and writing of the manuscript. SR participated in study design, performed the experiments, and participated in collection of data and writing of the manuscript. BS participated in the study design, statistical analysis and writing of the manuscript. MK participated in study design, collection of data, interpretation of the results and in writing the manuscript. SK performed the experiments, interpretation of the study results, and writing of the manuscript. All authors read and approved the final manuscript. All authors confirm authenticity all of the raw data.

\section{Ethics approval and consent to participate}

The animal protocols used in the present study were approved by the Institutional Animal Care and Use Committee (IACUC) at the University of Arkansas for Medical Sciences (approval no. 3882).

\section{Patient consent for publication}

Not applicable.

\section{Competing interests}

The authors declare that they have no competing interests.

\section{References}

1. Centers for Disease Control and Prevention: Adult Obesity Facts. CDC, 2020

2. World Health Oraganization: Overweight and obesity. WHO, 2020.

3. Ogden CL, Carroll MD, Curtin LR, Lamb MM and Flegal KM: Prevalence of high body mass index in US children and adolescents, 2007-2008. JAMA 303: 242-249, 2010.

4. Ogden CL, Carroll MD, Lawman HG, FryarCD, Kruszon-Moran D, Kit BK and Flegal KM: Trends in obesity prevalence among children and adolescents in the United States, 1988-1994 through 2013-2014. JAMA 315: 2292-2299, 2016.

5. Lazo M, Hernaez R, Eberhardt MS, Bonekamp S, Kamel I, Guallar E, Koteish A, Brancati FL and Clark JM: Prevalence of nonalcoholic fatty liver disease in the United States: The Third National Health and Nutrition Examination Survey, 1988-1994. Am J Epidemiol 178: 38-45, 2013. 
6. Angulo P: GI epidemiology: Nonalcoholic fatty liver disease. Aliment Pharmacol Ther 25: 883-889, 2007.

7. Welsh JA, Karpen S and Vos MB: Increasing prevalence of nonalcoholic fatty liver disease among United States adolescents, 1988-1994 to 2007-2010. J Pediatr 162: 496-500.e1, 2013.

8. Hakkak R, Al-Dwairi A, Fuchs GJ, Korourian S and Simmen FA: Dietary soy protein induces hepatic lipogenic enzyme gene expression while suppressing hepatosteatosis in obese female Zucker rats bearing DMBA-initiated mammary tumors. Genes Nutr 7: 549-558, 2012

9. Hakkak R, Zeng H, Dhakal IB and Korourian S: Short- and long-term soy diet versus casein protects liver steatosis independent of the arginine content. J Med Food 18: 1274-1280, 2015

10. Thomas I and Gregg B: Metformin; a review of its history and future: From lilac to longevity. Pediatr Diabetes 18: 10-16, 2017.

11. Khokhar A, Umpaichitra V, Chin VL and Perez-Colon S: Metformin use in children and adolescents with prediabetes Pediatr Clin North Am 64: 1341-1353, 2017.

12. Luong DQ, Oster R and Ashraf AP: Metformin treatment improves weight and dyslipidemia in children with metabolic syndrome. J Pediatr Endocrinol Metab 28: 649-655, 2015.

13. Madiraju AK, Qiu Y, Perry RJ, Rahimi Y, Zhang XM, Zhang D, Camporez JG, Cline GW, Butrico GM,Kemp BE, et al: Metformin inhibits gluconeogenesis via a redox-dependent mechanism in vivo. Nat Med 24: 1384-1394, 2018.

14. Lin HZ, Yang SQ, Chuckaree C, Kuhajda F, Ronnet G and Diehl AM: Metformin reverses fatty liver disease in obese, leptindeficient mice. Nat Med 6: 998-1003, 2000.

15. Marchesini G, Brizi M, Bianchi G, Tomassetti S, Zoli M and Melchionda N: Metformin in non-alcoholic steatohepatitis. Lancet 358: 893-894, 2001.

16. Xu H, Zhou Y, Liu Y, Ping J, Shou Q, Chen F and Ruo R: Metformin improves hepatic IRS2/PI3K/Akt signaling in insulin-resistant rats of NASH and cirrhosis. J Endocrinol 229: $133-144,2016$

17. Tiikkainen M, Häkkinen AM, Korsheninnikova E, Nyman T, Mäkimattila S and Yki-Järvinen H: Effects of rosiglitazone and metformin on liver fat content, hepatic insulin resistance, insulin clearance, and gene expression in adipose tissue in patients with type 2 diabetes. Diabetes 53: 2169-2176, 2004.

18. Nair S, Diehl AM, Wiseman M, Farr GH Jr and Perrillo RP: Metformin in the treatment of non-alcoholic steatohepatitis: A pilot open label trial. Aliment Pharmacol Ther 20: 23-28, 2004.

19. Lavine JE, Schwimmer JB, Van Natta ML, Molleston JP, Murray KF, Rosenthal P, Abrams SH, Scheimann AO, Sanyal AJ, Chalasani N, et al; Nonalcoholic Steatohepatitis Clinical Research Network: Effect of vitamin E or metformin for treatment of nonalcoholic fatty liver disease in children and adolescents: The TONIC randomized controlled trial. JAMA 305: 1659-1668, 2011.

20. Labruna G, Pasanisi F, Nardelli C, Caso R, Vitale DF, Contaldo F and Sacchetti L: High leptin/adiponectin ratio and serum triglycerides are associated with an 'at-risk' phenotype in young severely obese patients. Obesity (Silver Spring) 19: 1492-1496, 2011.

21. Korourian S, Hakkak R, Ronis MJJ, Shelnutt SR, Waldron J, Ingelman-Sundberg $M$ and Badger TM: Diet and risk of ethanol-induced hepatotoxicity: Carbohydrate-fat relationships in rats. Toxicol Sci 47: 110-117, 1999.

22. Zucker LM: Fat mobilization in vitro and in vivo in the genetically obese Zucker rat 'fatty'. J Lipid Res 13: 234-243, 1972.
23. Zucker LM and Zucker TF: Fatty, a new mutation in the rat. J Hered 52: 275-278, 1961.

24. Hakkak R, Gauss CH, Bell A and Korourian S: Short-term soy protein isolate feeding prevents liver steatosis and reduces serum ALT and AST levels in obese female zucker rats. Biomedicines 6: 6, 2018.

25. Zheng J, Woo SL, Hu X, Botchlett R, Chen L, Huo Y and Wu C: Metformin and metabolic diseases: A focus on hepatic aspects. Front Med 9: 173-186, 2015

26. Lu CH, Hung YJ and Hsieh PS: Additional effect of metformin and celecoxib against lipid dysregulation and adipose tissue inflammation in high-fat fed rats with insulin resistance and fatty liver. Eur J Pharmacol 789: 60-67, 2016.

27. Srinivasan S, Ambler GR, Baur LA, Garnett SP, Tepsa M, Yap F, Ward GM and Cowell CT: Randomized, controlled trial of metformin for obesity and insulin resistance in children and adolescents: Improvement in body composition and fasting insulin. J Clin Endocrinol Metab 91: 2074-2080, 2006.

28. Love-Osborne K, Sheeder J and Zeitler P: Addition of metformin to a lifestyle modification program in adolescents with insulin resistance. J Pediatr 152: 817-822, 2008.

29. Burgert TS, Duran EJ, Goldberg-Gell R, Dziura J, Yeckel CW, Katz S, Tamborlane WV and Caprio S: Short-term metabolic and cardiovascular effects of metformin in markedly obese adolescents with normal glucose tolerance. Pediatr Diabetes 9: 567-576, 2008.

30. Wilson DM, Abrams SH, Aye T, Lee PD, Lenders C, Lustig RH, Osganian SV and Feldman HA; Glaser Pediatric Research Network Obesity Study Group: Metformin extended release treatment of adolescent obesity: A 48-week randomized, double-blind, placebo-controlled trial with 48-week follow-up. Arch Pediatr Adolesc Med 164: 116-123, 2010.

31. Kay JP, Alemzadeh R, Langley G, D'Angelo L, Smith P and Holshouser S: Beneficial effects of metformin in normoglycemic morbidly obese adolescents. Metabolism 50: 1457-1461, 2001.

32. Atabek ME and Pirgon O: Use of metformin in obese adolescents with hyperinsulinemia: A 6-month, randomized, double-blind, placebo-controlled clinical trial. J Pediatr Endocrinol Metab 21: 339-348, 2008

33. El-Lakkany NM, Seif El-Din SH, Sabra AA, Hammam OA and Ebeid FA: Co-administration of metformin and $\mathrm{N}$-acetylcysteine with dietary control improves the biochemical and histological manifestations in rats with non-alcoholic fatty liver. Res Pharm Sci 11: 374-382, 2016

34. Ghadge A, Harsulkar A, Karandikar M, Pandit V and Kuvalekar A: Comparative anti-inflammatory and lipid-normalizing effects of metformin and omega-3 fatty acids through modulation of transcription factors in diabetic rats. Genes Nutr 11: 10, 2016.

35. Sui Y, Kong X, Fan R, Ye Y, Mai H, Zhuo S, Lu W, Ruan P, Fang $S$ and Yang T: Long-term treatment with metformin in the prevention of fatty liver in Zucker diabetic fatty rats. Diabetol Metab Syndr 11: 94, 2019.

36. Chen D, Jia D, Wu X, Shi K, Ren C, Dou Y, Guo M, Wang J, Ma M, $\mathrm{Wu} \mathrm{Z}$, et al: A novel metformin derivative showed improvement of lipid metabolism in obese rats with type 2 diabetes. Clin Exp Pharmacol Physiol 47: 1382-1392, 2020.

This work is licensed under a Creative Commons Attribution-NonCommercial-NoDerivatives 4.0 International (CC BY-NC-ND 4.0) License. 